\title{
UNIVERSAL ENVELOPING ALGEBRAS OF PBW TYPE*
}

\author{
ALESSANDRO ARDIZZONI \\ University of Ferrara, Department of Mathematics, Via Machiavelli 35, Ferrara I-44121, Italy \\ e-mail: alessandro.ardizzoni@unife.it
}

(Received 26 August 2010; revised 23 December 2010; accepted 11 April 2011; first published online 2 August 2011)

\begin{abstract}
We continue our investigation of the general notion of universal enveloping algebra introduced in [A. Ardizzoni, A Milnor-Moore type theorem for primitively generated braided Bialgebras, J. Algebra 327(1) (2011), 337-365]. Namely, we study a universal enveloping algebra when it is of Poincaré-Birkhoff-Witt (PBW) type, meaning that a suitable PBW-type theorem holds. We discuss the problem of finding a basis for a universal enveloping algebra of PBW type: as an application, we recover the PBW basis both of an ordinary universal enveloping algebra and of a restricted enveloping algebra. We prove that a universal enveloping algebra is of PBW type if and only if it is cosymmetric. We characterise braided bialgebra liftings of Nichols algebras as universal enveloping algebras of PBW type.
\end{abstract}

\section{Mathematics Subject Classification. Primary 16W30, Secondary 16S30.}

1. Introduction. Let $L$ be a Lie algebra which is assumed to have a totally ordered basis $(X, \leq)$. A classical result from the theory of Lie algebras asserts that the elements $x_{1}, \ldots, x_{n}$, where $n \geq 1, x_{i} \in X$, for all $1 \leq i \leq n$, and $x_{1} \leq x_{2} \leq \cdots \leq x_{n}$, along with 1 , form a basis of the universal enveloping algebra $U(L)$ of $L$. This theorem is due to Poincaré, Birkhoff and Witt and the basis is called the PBW basis of the universal enveloping algebra (see e.g. [15, Corollary C, p. 92]). This result essentially relies on the existence of a bialgebra map $\omega: S(L) \rightarrow \mathfrak{G}(U(L))$, where $S(L)$ denotes the symmetric algebra on $L$ and $\mathfrak{G}(U(L))$ is the graded braided bialgebra associated with the standard filtration of $U(L)$. The fact that the map $\omega$ is bijective sometimes is called the PBW theorem, see e.g. [15, Corollary C, p. 92].

Motivated by these observations, we intend to investigate a PBW-type theorem for a general notion of universal enveloping algebra appeared in [2, Definition 5.2]. To explain better this notion we need to recall the definition of braided bialgebra.

Recall that a braided vector space $(V, c)$ consists of a vector space $V$ and a $K$ linear map $c: V \otimes V \rightarrow V \otimes V$, called braiding, obeying the so-called quantum YangBaxter equation $c_{1} c_{2} c_{1}=c_{2} c_{1} c_{2}$. Here $c_{1}=c \otimes V$ and $c_{2}=V \otimes c$. A braided bialgebra is then a braided vector space which is both an algebra and a coalgebra with structures suitably compatible with the braiding. Examples of braided bialgebras are all bialgebras in those braided monoidal categories which are monoidal subcategories of the category of vector spaces.

In [2, Theorem 6.9], it is proved that every primitively generated braided bialgebra is isomorphic, as a braided bialgebra, to the generalised universal enveloping algebra

*This paper was written while the author was member of GNSAGA with partial financial support from MIUR within the National Research Project PRIN 2007 
$U(V, c, b)$ of its infinitesimal braided Lie algebra $(V, c, b)$ (here $b$ denotes the bracket on the braided vector space $(V, c)$ consisting of primitive elements in the given braided bialgebra). This result can be seen as an extension of the celebrated Milnor-Moore theorem [23, Theorem 5.18] for cocommutative connected bialgebras (once observed that such a bialgebra is always primitively generated): in characteristic zero, any cocommutative connected bialgebra is the enveloping algebra of its space of primitive elements, regarded as a Lie algebra in a canonical way.

Now, in order to investigate a PBW-type theorem for $U(V, c, b)$, we have to choose an appropriate substitute for the symmetric algebra. Since in the classical case such an algebra is obtained as the universal enveloping algebra of $L$ regarded as a Lie algebra through the trivial bracket, the natural candidate is the Nichols algebra $\mathcal{B}(V, c)$ which is indeed of the form $U\left(V, c, b_{t r}\right)$, where $b_{t r}$ denotes the trivial bracket on $(V, c)$.

The results in Detail. The paper is organised as follows: Section 2 contains preliminary facts and notations that will be used in the paper; Section 3 deals with the standard filtration $\left(U_{(n)}\right)_{n \in \mathbb{N}}$ on $U:=U(V, c, b)$. This filtration is induced by the standard filtration on the braided tensor algebra $T(V, c)$ (the latter is just the tensor algebra $T(V)$ which is regarded as a braided bialgebra through a comultiplication depending on the braiding $c$ ). Having in mind the classical case, we consider the graded braided bialgebra $\mathfrak{G}(U)$ associated with the standard filtration. Denote by $\operatorname{gr} U$ the graded braided bialgebra associated with the coradical filtration of $U$. In Proposition 3.6, we produce a graded braided bialgebra homomorphism $\xi_{U}: \mathfrak{G}(U) \rightarrow \operatorname{gr} U$ and we characterise when this morphism is bijective. In Proposition 3.7 and Proposition 3.8, we show there exist canonical graded braided bialgebra homomorphisms $\vartheta_{U}$ : $T(V, c) \rightarrow \mathfrak{G}(U)$ and $\chi_{U}: \mathcal{B}(V, c) \rightarrow \operatorname{gr} U$ such that the diagram

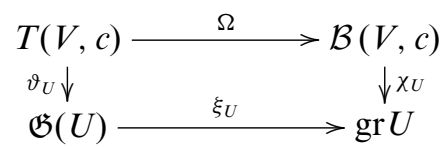

commutes, where $\Omega$ denotes the canonical projection. Moreover, $\vartheta_{U}$ is surjective and $\chi_{U}$ is injective.

We say that $U$ is of $P B W$ type whenever $\vartheta_{U}$ quotients to a braided bialgebra isomorphism $\omega_{U}: \mathcal{B}(V, c) \rightarrow \mathfrak{G}(U)$ :

$$
T(V, c) \underset{\vartheta_{U}-\mathcal{G}(U)}{\stackrel{\Omega}{L \omega_{U}}} \longrightarrow \mathcal{B}(V, c)
$$

Therefore, $U$ is of PBW type means that $U$ fulfills a PBW-type theorem. In Theorem 3.11 , we prove that $U(V, c, b)$ is always of $\mathrm{PBW}$ type whenever $(V, c)$ has combinatorial rank at most one in the sense of [19, Definition 5.4].

Section 4 deals with the problem of determining a basis for a universal enveloping algebra. In Proposition 4.2, mimicking classical ideas, we find a criterion that helps to obtain a basis of $U=U(V, c, b)$ knowing a suitable basis of $\mathcal{B}(V, c)$ in case $U$ is of PBW type. This criterion is applied in Example 4.4 and Example 4.6 to recover the PBW basis both of an ordinary universal enveloping algebra and of a restricted enveloping algebra. In Example 4.7, we compute a PBW basis for the universal enveloping algebra of a braided Lie algebra whose bracket $c$ is not a symmetry, i.e. $c^{2} \neq \mathrm{Id}$. 
Section 5, is devoted to the investigation of universal enveloping algebras which are cosymmetric in the sense of [20, Definition 3.1]. In Theorem 5.2, using the results in [20], we give several characterisations of the fact that $U$ is of PBW type: in particular, this is equivalent to require that $U$ is cosymmetric. In Theorem 5.4, we provide a sufficient condition to have that $U$ is cosymmetric. This result is used in Corollary 5.5 to get that a braided vector space has combinatorial rank, at most, $n+1$ whenever the corresponding symmetric algebra of rank $n$ is cosymmetric. As a result, in Example 5.6, we exhibit a universal enveloping algebra $U$ which is not of PBW type.

In Section 6, we investigate braided bialgebra liftings of the Nichols algebra. Explicitly, given a braided vector space $(V, c)$, we say that a braided bialgebra $B$ is a lifting of $\mathcal{B}(V, c)$ if there is a graded braided bialgebra isomorphism $\mathcal{B}(V, c) \cong \operatorname{gr} B$. Theorem 6.2 characterises braided bialgebra liftings of Nichols algebras as universal enveloping algebras of PBW type. This result is applied in Corollary 6.3 to Nichols algebras algebras of a braided vector space of combinatorial rank at most one.

On PBW-type theorems. Several attempts to extend the classical PBW results to more general contexts appeared in the literature. Some of them, such as $[\mathbf{2 1}, \mathbf{2 6}, \mathbf{2 7}, \mathbf{3 3}]$, are related the quantised enveloping algebras $U_{q}(\mathfrak{g})$ of Drinfeld and Jimbo (note that this enveloping algebra is pointed but not connected whence it can not be described as a universal enveloping algebra of our kind). We now list some PBW-type results which are closer to our approach (see also the references therein):

- A PBW Theorem for connected braided Hopf algebras with involutive braidings was obtained in [20, Theorem 7.1]. Our result can be seen as an extension of this one to the non-symmetric case.

- A PBW Theorem for quadratic algebras can be found in [9] and in [10]. See also [7, Theorem 3.9].

- Deep results on the PBW basis are obtained in [18, Theorem 2] and, more generally, in [30, Theorem 34]) for braided vector spaces of diagonal type or left triangular, respectively. See also the more recent paper [14].

We would like to point out that our aim here is not to compute explicitly a PBW basis for the Nichols algebra associated with a braided vector space. Instead, we will give a method to produce a basis for a universal enveloping algebra $U(V, c, b)$ of PBW type once known a basis for the Nichols algebra $\mathcal{B}(V, c)$, see Remark 4.3.

2. Preliminaries. Throughout this paper, $K$ will denote a field. All vector spaces will be defined over $K$ and the tensor product over $K$ will be denoted by $\otimes$.

In this section, we recall the main notions that we will deal with in the paper.

Definition 2.1. Let $V$ be a vector space over a field $K$. A $K$-linear map $c=c_{V}$ : $V \otimes V \rightarrow V \otimes V$ is called a braiding if it satisfies the quantum Yang-Baxter equation $c_{1} c_{2} c_{1}=c_{2} c_{1} c_{2}$ on $V \otimes V \otimes V$, where we set $c_{1}:=c \otimes V$ and $c_{2}:=V \otimes c$. The pair $(V, c)$ will be called a braided vector space. A morphism of braided vector spaces $\left(V, c_{V}\right)$ and $\left(W, c_{W}\right)$ is a $K$-linear map $f: V \rightarrow W$ such that $c_{W}(f \otimes f)=(f \otimes f) c_{V}$.

A general method for producing braided vector spaces is to take an arbitrary braided category $(\mathcal{M}, \otimes, K, a, l, r, c)$, which is a monoidal subcategory of the category Vect $_{K}$ of $K$-vector spaces (here $a, l, r$ denote the associativity, the left and the right unit constraints, respectively). Hence any object $V \in \mathcal{M}$ can be regarded as a braided vector 


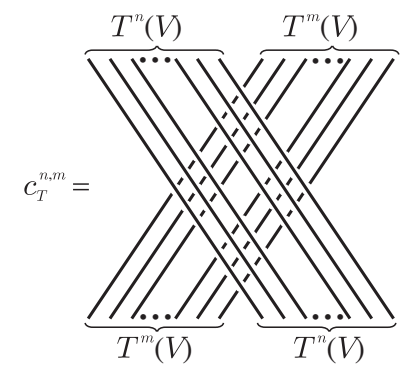

Figure 1 The braiding $c_{T}$.

space with respect to $c:=c_{V, V}$, where $c_{X, Y}: X \otimes Y \rightarrow Y \otimes X$ denotes the braiding in $\mathcal{M}$, for all $X, Y \in \mathcal{M}$.

Let $\mathcal{N}$ be either the category of comodules over a coquasitriangular Hopf algebra or the category of Yetter-Drinfeld modules over a Hopf algebra with bijective antipode. Then the forgetful functor $F: \mathcal{N} \rightarrow$ Vect $_{K}$ is a strict monoidal functor. Hence $\mathcal{M}=$ $\operatorname{Im} F$ is an example of a category as above.

Definition 2.2. [8] A quadruple $(A, m, u, c)$ is called a braided algebra if $(A, m, u)$ is an associative unital algebra and $(A, c)$ is a braided vector space for which the following conditions hold:

$$
\begin{aligned}
c(m \otimes A)=(A \otimes m)(c \otimes A)(A \otimes c), & c(A \otimes m)=(m \otimes A)(A \otimes c)(c \otimes A), \\
c(u \otimes A)=A \otimes u, & c(A \otimes u)=u \otimes A .
\end{aligned}
$$

A morphism of braided algebras is, by definition, a morphism of ordinary algebras which, in addition, is a morphism of braided vector spaces. Similarly, the notions of braided coalgebra and of morphism of braided coalgebras is introduced.

[29, Definition 5.1] A sextuple $(B, m, u, \Delta, \varepsilon, c)$ is a called a braided bialgebra if $(B, m, u, c)$ is a braided algebra, $(B, \Delta, \varepsilon, c)$ is a braided coalgebra and the following relation hold:

$$
\Delta m=(m \otimes m)(B \otimes c \otimes B)(\Delta \otimes \Delta) .
$$

Examples of the notions above are algebras, coalgebras and bialgebras in any braided category $\mathcal{M}$ which is a monoidal subcategory of Vect $_{K}$. The notion of braided bialgebra admits a graded counterpart which is called graded braided bialgebra. For further results on this topic, the reader is refereed to $[5,1.8]$.

EXAMPLE 2.3. Let $(V, c)$ be a braided vector space. Consider the tensor algebra $T=T(V)$ with multiplication $m_{T}$ and unit $u_{T}$. This is a graded braided algebra with $n$th graded component $T^{n}(V)=V^{\otimes n}$. The braiding $c_{T}$ on $T$ is defined using the braiding $c$ of $V$ (the graded component $c_{T}^{n, m}$ of $c_{T}$ is represented in Figure 1, where each crossing stands for a copy of $c$ ).

Now $T \otimes T$ becomes itself an algebra with multiplication $m_{T \otimes T}:=$ $\left(m_{T} \otimes m_{T}\right)\left(T \otimes c_{T} \otimes T\right)$. This algebra is denoted by $T \otimes_{c} T$. The universal property of the tensor algebra yields two algebra homomorphisms $\Delta_{T}: T \rightarrow T \otimes_{c} T$ and $\varepsilon_{T}: T \rightarrow K$. It is straightforward to check that $\left(T, m_{T}, u_{T}, \Delta_{T}, \varepsilon_{T}, c_{T}\right)$ is a graded 
braided bialgebra. Note that $\Delta_{T}$ really depends on $c$. For example, one has $\Delta_{T}(z)=$ $z \otimes 1+1 \otimes z+(c+\mathrm{Id})(z)$, for all $z \in V \otimes V$.

DEFINITION 2.4. The graded braided bialgebra described in Example 2.3 is called the braided tensor algebra and will be denoted by $T(V, c)$.

Claim 2.5. Recall that a coalgebra $C$ is called connected if the coradical $C_{0}$ of $C$ (i.e. the sum of all simple subcoalgebras of $C$ ) is one-dimensional. In this case, there is a unique group-like element $1_{C} \in C$ such that $C_{0}=K 1_{C}$. A morphism of connected coalgebras is just a coalgebra homomorphisms (clearly, it preserves the group-like element).

By definition, a braided coalgebra $(C, c)$ is connected if the underlying coalgebra is connected and, for any $x \in C, c\left(x \otimes 1_{C}\right)=1_{C} \otimes x$ and $c\left(1_{C} \otimes x\right)=x \otimes 1_{C}$.

DEFINITION 2.6.

(1) Let $B$ be a braided bialgebra with comultiplication $\Delta$ and unit $1_{B}$. Consider the space

$$
P(B)=\left\{b \in B \mid \Delta(b)=b \otimes 1_{B}+1_{B} \otimes b\right\}
$$

of primitive elements in $B$. By [3, Lemma 2.10], the braiding of $B$ induces a braiding $c_{P}$ of $P$ that will be called the infinitesimal braiding of $B$. The braided vector space $\left(P, c_{P}\right)$ will be called the infinitesimal part of $B$ (see [3, Definition 2.11]).

(2) A braided bialgebra $B$ is called primitively generated if it is generated as an algebra by $P(B)$. See [23, p. 239].

REMARK 2.7.

(1) Let $B$ be a primitively generated braided bialgebra. Then the underlying braided coalgebra is connected (see [3, Proposition 5.8]).

(2) Let $B$ be a connected braided bialgebra. Since $B_{0}=K 1_{B}$, then $\operatorname{Id}_{B}$ is convolution invertible in $\operatorname{Hom}\left(B_{0}, B\right)$. In view of the Takeuchi's result [24, Lemma 5.2.10], we conclude that $\operatorname{Id}_{B}$ is convolution invertible in $\operatorname{Hom}(B, B)$. Hence $B$ has an antipode, i.e. it is a braided Hopf algebra (this reasoning is similar to [1, Remark 9.17] which is due to Masuoka). In particular, by (1), any primitively generated braided bialgebra is indeed a braided Hopf algebra.

Definition 2.8. [2, Definitions 5.2 and 5.4] Let $(V, c)$ be a braided vector space. Suppose that, for each $n \in \mathbb{N}$, there are a braided bialgebra $U^{[n]}$ and a map $i^{[n]}$ : $V \rightarrow P\left(U^{[n]}\right)$ that fulfil the following requirements, where we set $P^{[n]}:=P\left(U^{[n]}\right)$ and $V^{[n]}:=i^{[n]}(V)$.

- $U^{[0]}:=T(V, c)$ and $i^{[0]}: V \rightarrow P^{[0]}$ is the restriction of the canonical map $V \rightarrow$ $U^{[0]}$.

- For each $n \in \mathbb{N}$, there exists a map $b^{[n]}: P^{[n]} \rightarrow V$ such that

$$
\begin{aligned}
& c_{V^{[n]}}\left(i^{[n]} b^{[n]} \otimes V^{[n]}\right)=\left(V^{[n]} \otimes i^{[n]} b^{[n]}\right) c_{P^{[n]}, V^{[n]}}, \\
& c_{V^{[n]}}\left(V^{[n]} \otimes i^{[n]} b^{[n]}\right)=\left(i^{[n]} b^{[n]} \otimes V^{[n]}\right) c_{V^{[n]}, P^{[n]}},
\end{aligned}
$$

where $c_{V^{[n]}}: V^{[n]} \otimes V^{[n]} \rightarrow V^{[n]} \otimes V^{[n]}$ and $c_{P^{[n]}, V^{[n]}},: P^{[n]} \otimes V^{[n]} \rightarrow V^{[n]} \otimes P^{[n]}$ denote the restrictions of the braiding of $P^{[n]}$. 
- For each $n \in \mathbb{N}$,

$$
U^{[n+1]}=\frac{U^{[n]}}{\left(\left[\operatorname{Id}-i^{[n]} b^{[n]}\right]\left[P^{[n]}\right]\right)},
$$

$\pi_{n}^{n+1}: U^{[n]} \rightarrow U^{[n+1]}$ is the natural projection of $U^{[n]}$ onto $U^{[n+1]}$ and $i^{[n+1]}$ :

$V \rightarrow P^{[n+1]}$ is the restriction of the canonical map $V \rightarrow U^{[n+1]}$, so that $i^{[n+1]}=$ $P\left(\pi_{n}^{n+1}\right) \circ i^{[n]}$, where $P\left(\pi_{n}^{n+1}\right): P^{[n]} \rightarrow P^{[n+1]}$ is the map induced by $\pi_{n}^{n+1}$.

In this case we will say that $b:=\left(b^{[n]}\right)_{n \in \mathbb{N}}$ is a bracket for a braided vector space $(V, c)$. This way, we get a direct system of braided bialgebras

$$
U^{[0]} \stackrel{\pi_{0}^{1}}{\rightarrow} U^{[1]} \stackrel{\pi_{1}^{2}}{\rightarrow} U^{[2]} \stackrel{\pi_{2}^{3}}{\rightarrow} \cdots
$$

The direct limit of this direct system will be denoted by $\left(U(V, c, b), \pi_{U}\right):=$ $\left(U^{[\infty]}, \pi_{n}^{\infty}\right)$, where $\pi_{U}: T(V, c) \rightarrow U(V, c, b)$ is the canonical projection. Now $U(V, c, b)$ becomes a primitively generated braided bialgebra which will be called the universal enveloping algebra of $(V, c, b)$. Denote by $i_{U}: V \rightarrow U(V, c, b)$ the (not necessarily injective) canonical map and set $P^{[\infty]}:=P\left(U^{[\infty]}\right)$. Note that $\operatorname{Im}\left(i_{U}\right) \subseteq P^{[\infty]}$ so that $i_{U}$ induces a morphism of braided vector spaces $i^{[\infty]}: V \rightarrow P^{[\infty]}$.

We say that $(V, c, b)$ is a braided Lie algebra whenever $(V, c)$ is a braided vector space, $b$ is a bracket on $(V, c)$ and $i_{U}: V \rightarrow U(V, c, b)$ is injective.

Let $B$ be a braided bialgebra. By [2, Theorem 6.5], the infinitesimal part $\left(P, c_{P}\right)$ of $B$ is endowed with a specific bracket $b_{P}$ such that $\left(P, c_{P}, b_{P}\right)$ is a braided Lie algebra. $\left(P, c_{P}, b_{P}\right)$ will be called the infinitesimal braided Lie algebra of $B$.

REMARK 2.9.

(1) Let $(V, c, b)$ be a braided Lie algebra and set $U:=U(V, c, b)$. In view of [2, Corollary 5.6], the map $i_{U}: V \rightarrow U$ induces an isomorphism between $V$ and $P(U)$.

(2) By [2, Theorem 6.9], every primitively generated braided bialgebra is isomorphic as a braided bialgebra to the universal enveloping algebra of its infinitesimal braided Lie algebra.

REMARK 2.10. Let $(V, c, b)$ be a braided Lie algebra. When $c$ is a symmetry, i.e. $c^{2}=\operatorname{Id}_{V \otimes V}$, and the characteristic of $K$ is zero, our universal enveloping algebra $U(V, c, b)$ reduces to the one introduced in [12] (cf. [3, Remark 6.4] using Remark 3.10 below). Other notions of Lie algebra and universal enveloping algebra, extending the ones in [12] to the non-symmetric case, appeared in the literature. Let us mention some of them without any pretension of exhaustiveness:

- Lie algebras for braided vector spaces $(V, c)$ where $c$ is a braiding of Hecke type [31, Definition 7.1]. Compare with [3, Section 6] using Remark 3.10 to see how our notion of universal enveloping algebra behaves in this setting.

- Lie algebras for braided vector spaces $(V, c)$ where $c$ is constructed by means of braidings of Hecke type [13, Definition 1]. See [7, Remark 3.6] using Remark 3.10 .

- Braided Lie algebras for objects $L$ in a braided or quasi-tensor category equipped with a co-product, a co-unit and a bracket [,]: $L \otimes L \rightarrow L$ satisfying some axioms, [22, Definition 4.1]. Here the universal enveloping algebra is defined as the quotient of the tensor algebra over $L$ modulo some quadratic relations. 
- Quantum Lie algebras for objects $\mathfrak{g}$ in a monoidal category equipped with a braiding $\sigma: \mathfrak{g} \otimes \mathfrak{g} \rightarrow \mathfrak{g} \otimes \mathfrak{g}$ and a bracket [,]: $\mathfrak{g} \otimes \mathfrak{g} \rightarrow \mathfrak{g}$ satisfying some axioms, see [11, Definition 2.1], which naturally arises in the context of covariant differential calculus over quantum groups [32, Theorems 5.3 and 5.4].

- Lie algebras for objects in the braided monoidal category of Yetter-Drinfeld modules over a Hopf algebra with bijective antipode [25, Definition 4.1]. Compare with [3, Section 8] using Remark 3.10.

- Lie algebras defined by considering quantum operations (see [17, Definition 2.2]) as primitive polynomials in the tensor algebra. When the underline braided vector space is an object in the category of Yetter-Drinfeld modules over some group algebra, to any Lie algebra of this kind, Kharchenko associates a universal enveloping algebra which is not connected (see [19]). Note that the universal enveloping algebra we deal with in the present paper is connected as it is meant to describe the structure of primitively generated (whence connected) braided bialgebras over $K$, see (2) in Remark 2.9.

DEFINITION 2.11. In view of [2, Example 7.1], any braided vector space $(V, c)$ can be endowed with the so-called trivial bracket $b_{t r}$, which makes of $\left(V, c, b_{t r}\right)$ a braided Lie algebra. Set $U:=U\left(V, c, b_{t r}\right)$. Then $U^{[n]}$ is called the symmetric algebra of rank $n$ of $(V, c)$ and is denoted by $S^{[n]}$. It is the braided bialgebra $S^{[n]}(B)$ introduced in [4, Definition 3.10] in the case $B=T(V, c)$. Explicitly, $S^{[0]}=T(V, c)$ and, for all $n>0$, $S^{[n]}$ is the graded braided bialgebra obtained dividing out $S^{[n-1]}$ by the two-sided ideal generated by the homogeneous primitive elements in $S^{[n-1]}$ of degree at least two. Moreover, $U^{[\infty]}=U$ is denoted by $S^{[\infty]}$ and identifies with the Nichols algebra $\mathcal{B}(V, c)$ (see $[4,5.3]$ for a different definition). We will denote by $\Omega: T(V, c) \rightarrow \mathcal{B}(V, c)$ the canonical projection.

If there exists a least $n \in \mathbb{N}$ such that $S^{[n]}=S^{[\infty]}$, then we will say that $(V, c)$ has combinatorial rank $n$ (cf. [19, Definition 5.4], see also [4, Section 5]). 5.4 .

We include here a technical result about $P^{[n]}$ that will be used to prove Theorem

LEMMA 2.12. Let $(V, c, b)$ be a braided Lie algebra. Let $P^{[n]}$ and $V^{[n]}$ be the spaces introduces in Definition 2.8. Set $W^{[n]}:=\operatorname{Ker}\left(\pi_{n}^{n+1}\right) \cap P^{[n]}$. Then $W^{[n]}=\operatorname{Ker}\left(b^{[n]}\right)=$ $\operatorname{Im}\left(\operatorname{Id}_{P^{[n]}}-i^{[n]} b^{[n]}\right)$ and $P^{[n]}=W^{[n]} \oplus V^{[n]}$, as a direct sum of braided subspaces.

Proof. As in Definition 2.8, denote by $P\left(\pi_{n}^{n+1}\right): P^{[n]} \rightarrow P^{[n+1]}$ the natural map induced by $\pi_{n}^{n+1}$. Since $(V, c, b)$ is a braided Lie algebra, by [2, Proposition 5.7], we have that $b^{[n+1]} P\left(\pi_{n}^{n+1}\right)=b^{[n]}$ and $b^{[n]} i^{[n]}=\operatorname{Id}_{P^{[n]}}$. By the first equality, for $w \in W^{[n]}$, we have

$$
0=b^{[n+1]} \pi_{n}^{n+1}(w) \stackrel{w \in P^{[n]}}{=} b^{[n+1]} P\left(\pi_{n}^{n+1}\right)(w)=b^{[n]}(w),
$$

so that $W^{[n]} \subseteq \operatorname{Ker}\left(b^{[n]}\right)$. On the other hand, if $z \in \operatorname{Ker}\left(b^{[n]}\right)$, then $z \in P^{[n]}$ and

$$
\pi_{n}^{n+1}(z) \stackrel{z \in P^{[n]}}{=} \pi_{n}^{n+1} i^{[n]} b^{[n]}(z)=0,
$$

so that $\operatorname{Ker}\left(b^{[n]}\right) \subseteq W^{[n]}$ whence $W^{[n]}=\operatorname{Ker}\left(b^{[n]}\right)$. Now, from $b^{[n]} i^{[n]}=\operatorname{Id}_{P^{[n]}}$, one gets $W^{[n]}=\operatorname{Ker}\left(b^{[n]}\right)=\operatorname{Im}\left(\operatorname{Id}_{P^{[n]}}-i^{[n]} b^{[n]}\right)$ and $P^{[n]}=W^{[n]} \oplus V^{[n]}$, as a direct sum of braided subspaces. 
Finally, for the reader's convenience, we quote a technical result we will invoke three times in the paper.

Lemma 2.13. [24, Lemma 5.3.3] If $C$ is connected and $f: C \rightarrow D$ is a coalgebra map such that $f_{\mid P(C)}$ is injective, then $f$ is injective.

3. Standard versus coradical filtration. In this section, we introduce the standard filtration of a universal enveloping algebra and we study it in connection with the coradical filtration of the underlying coalgebra.

DEFINITION 3.1. Recall that a filtration on a vector space $M$ is an increasing sequence $M_{(0)} \subseteq M_{(1)} \subseteq \cdots \subseteq M_{(n)} \subseteq \cdots$ of subspaces of $M$. By convention we write $M_{(-1)}:=0$. A filtration $\left(M_{(n)}\right)_{n \in \mathbb{N}}$ on a vector space $M$ gives rise to a graded module

$$
\mathfrak{G}(M):=\bigoplus_{n \in \mathbb{N}} \mathfrak{G}^{n}(M) \quad \text { where } \quad \mathfrak{G}^{n}(M):=\frac{M_{(n)}}{M_{(n-1)}} .
$$

Let $M$ and $N$ be filtered vector spaces with filtrations $\left(M_{(n)}\right)_{n \in \mathbb{N}}$ and $\left(N_{(n)}\right)_{n \in \mathbb{N}}$, respectively. A filtered homomorphism is a $K$-linear map $f: M \rightarrow N$ such that $f\left(M_{(n)}\right) \subseteq N_{(n)}$ for all $n \in \mathbb{N}$. Such a morphism induces in a natural way a graded homomorphism $\mathfrak{G}(f): \mathfrak{G}(M) \rightarrow \mathfrak{G}(N)$. The $n$th graded component of $\mathfrak{G}(f)$ will be denoted by $\mathfrak{G}^{n}(f)$.

A braided bialgebra $\left(B, c_{B}\right)$ is called filtered if the underlying vector space has a filtration $\left(B_{(n)}\right)_{n \in \mathbb{N}}$ with $B=\cup B_{(n)}$ such that

$$
\Delta_{B}\left(B_{(n)}\right) \subseteq \sum_{i=0}^{n} B_{(i)} \otimes B_{(n-i)}, \quad B_{(i)} \cdot B B_{(j)} \subseteq B_{(i+j)} \quad \text { and } \quad c_{B}\left(B_{(i)} \otimes B_{(j)}\right) \subseteq B_{(j)} \otimes B_{(i)}
$$

for all $i, j \in \mathbb{N}$. A filtered braided bialgebra homomorphism $f:\left(B, c_{B}\right) \rightarrow$ $\left(B^{\prime}, c_{B^{\prime}}\right)$ is a filtered homomorphism $f: B \rightarrow B^{\prime}$ which is also a braided bialgebra homomorphism.

LEMMA 3.2. Let $\left(B, c_{B}\right)$ be a filtered braided bialgebra with filtration $\left(B_{(n)}\right)_{n \in \mathbb{N}}$. Assume $B_{(0)}=K$. Then the space $\mathfrak{G}(B)$ is a graded braided bialgebra with structures induced by those of $B$. Furthermore, any filtered braided bialgebra homomorphism $f$ : $\left(B, c_{B}\right) \rightarrow\left(B^{\prime}, c_{B^{\prime}}\right)$ induces a graded braided bialgebra homomorphism $\mathfrak{G}(f): \mathfrak{G}(B) \rightarrow$ $\mathfrak{G}\left(B^{\prime}\right)$.

Proof. Set $G:=\mathfrak{G}(B), G^{n}:=B_{(n)} / B_{(n-1)}$ and let $p_{n}: B_{(n)} \rightarrow G^{n}$ be the canonical projection, for every $n \in \mathbb{N}$. Since $\left(B_{(n)}\right)_{n \in \mathbb{N}}$ is a coalgebra filtration on $B$, then $G$ carries a graded coalgebra structure $\left(G, \Delta_{G}, \varepsilon_{G}\right)$ (see [28, p. 230]). Moreover, the coradical of $B$ is contained in $B_{(0)}$ (see [28, Proposition 11.1.1]) whence it is $K$. In particular $1_{B} \in B_{(0)}$. Thus $\left(B_{(n)}\right)_{n \in \mathbb{N}}$ is also an algebra filtration on $B$ whence $G$ carries a graded algebra structure too (see [28, p. 230]). Since by definition $c_{B}\left(B_{(i)} \otimes B_{(j)}\right) \subseteq B_{(j)} \otimes B_{(i)}$ for all $i, j \in \mathbb{N}$, then $c_{B}$ induces a braiding $c_{G}^{a, b}: G^{a} \otimes G^{b} \rightarrow G^{b} \otimes G^{a}$ for all $a, b \in \mathbb{N}$. It is straightforward to prove that $\left(G, m_{G}, u_{G}, \Delta_{G}, \varepsilon_{G}, c_{G}\right)$ is indeed a graded braided bialgebra. Let $f:\left(B, c_{B}\right) \rightarrow\left(B^{\prime}, c_{B^{\prime}}\right)$ be a filtered braided bialgebra homomorphism. By [28, p. 229], $f$ induces a graded coalgebra map $\mathfrak{G}(f): \mathfrak{G}\left(B, c_{B}\right) \rightarrow \mathfrak{G}\left(B^{\prime}, c_{B^{\prime}}\right)$. Furthermore, the same map is also a graded algebra map. It is easy to check that $\mathfrak{G}(f)$ is also a morphism of braided vector spaces whence a graded braided bialgebra homomorphism.

Let $(V, c)$ be a braided vector space and set $T:=T(V, c)$. Recall that the standard filtration on $T$ is defined by setting $T_{(n)}:=\oplus_{i=0}^{n} V^{\otimes i}$. 
Lemma 3.3. Let $(V, c, b)$ be a braided bialgebra and set $U:=U(V, c, b)$. Let $\left(T_{(n)}\right)_{n \in \mathbb{N}}$ be the standard filtration on $T(V, c)$. Set $U_{(n)}:=\pi_{U}\left(T_{(n)}\right)$, for each $n \in \mathbb{N}$. Then $\left(U_{(n)}\right)_{n \in \mathbb{N}}$ is a filtration on $U$ that makes it a filtered connected braided bialgebra.

Proof. It is straightforward.

Definition 3.4. Let $(V, c, b)$ be a braided Lie algebra and set $U:=U(V, c, b)$.

The filtration $\left(U_{(n)}\right)_{n \in \mathbb{N}}$ of Lemma 3.3 will be called the standard filtration on $U$ and $\mathfrak{G}(U)$ will denote the graded braided bialgebra associated with this filtration.

We say that $U$ is strictly generated by $V$ whenever the standard and the coradical filtration on $U$ coincide. This means the $n$th term $U_{n}$ of the coradical filtration of $U$ is given by $U_{n}=U_{(n)}=\sum_{i=0}^{n} \pi_{U}(V)^{i}$.

REMARK 3.5. Let $B$ be a braided bialgebra. If $B$ is connected, then the coradical filtration $\left(B_{n}\right)_{n \in \mathbb{N}}$ of $B$ makes $B$ itself into a filtered connected braided bialgebra. Thus, by Lemma 3.2, the graded coalgebra $\operatorname{gr} B$ associated with the coradical filtration of $B$ is indeed a graded braided bialgebra. Since $B$ is connected, by [28, Proposition 11.1.1] we have that $\operatorname{gr} B$ is connected too. Thus, by [28, Lemma 11.2.3] $\operatorname{gr} B$ is strictly graded as a coalgebra. In particular, $P(\operatorname{gr} B)=\operatorname{gr}^{1} B=B_{1} / B_{0}$.

Proposition 3.6. Let $(V, c, b)$ be a braided Lie algebra and set $U:=U(V, c, b)$. Then the identity on $U$ induces a graded braided bialgebra homomorphism $\xi_{U}: \mathfrak{G}(U) \rightarrow \operatorname{gr} U$. The following assertions are equivalent:

(1) $\xi_{U}$ is bijective.

(2) $\xi_{U}$ is surjective.

(3) $\xi_{U}$ is injective.

(4) $U_{(n)} \cap U_{n-1}=U_{(n-1)}$ for all $n \in \mathbb{N}$.

(5) $U_{n}=U_{(n)}+U_{n-1}$ for all $n \in \mathbb{N}$.

(6) $U$ is strictly generated by $V$.

(7) $P(\mathfrak{G}(U))=U_{(1)} / U_{(0)}$.

(8) $\pi_{U}(V)^{n} \cap U_{n-1} \subseteq U_{(n-1)}$ for all $n \in \mathbb{N}$.

Proof. Note that, for each $n \in \mathbb{N}$, one has $U_{(n)} \subseteq U_{n}$ so that $\operatorname{Id}_{U}$ is a filtered braided bialgebra automorphism whence, by Lemma 3.2, it induces a graded braided bialgebra homomorphism $\xi_{U}: \mathfrak{G}(U) \rightarrow \operatorname{gr} U$.

(6) $\Rightarrow$ (1) $\Rightarrow$ (2) , (1) $\Rightarrow$ (3) These implications are trivial.

(3) $\Leftrightarrow$ (4) Let $\xi_{U}^{n}: U_{(n)} / U_{(n-1)} \rightarrow U_{n} / U_{n-1}$ be the $n$th graded component of $\xi_{U}$. Then $\operatorname{Ker}\left(\xi_{U}^{n}\right)=\left[U_{(n)} \cap U_{n-1}\right] / U_{(n-1)}$.

(4) $\Rightarrow(6)$ It is enough to prove that $U_{n} \subseteq U_{(n)}$. Let $z \in U_{n}$. Since $\pi_{U}(V)$ generates $U$ as a $K$-algebra, there is a least $t \in \mathbb{N}$ such that $z \in U_{(t)}$. If $t \geq n+1$, then $z \in$ $U_{(t)} \cap U_{n} \subseteq U_{(t)} \cap U_{t-1}=U_{(t-1)}$ contradicting the minimality of $t$. Then $t \leq n$ whence $z \in U_{(t)} \subseteq U_{(n)}$.

(2) $\Leftrightarrow$ (5) It follows from $\xi_{U}\left(U_{(n)} / U_{(n-1)}\right)=\left[U_{(n)}+U_{n-1}\right] / U_{n-1}$.

(5) $\Rightarrow$ (6) By induction on $n \in \mathbb{N}$, we deduce that $U_{n}=U_{(n)}$.

$(1) \Rightarrow(7)$ Since $U$ is connected, by Remark 3.5, the primitive part of $\operatorname{gr} U$ is $\operatorname{gr}^{1} U$. Since $\xi_{U}$ is a graded braided bialgebra isomorphism we get that $P(\mathfrak{G}(U))=\mathfrak{G}^{1}(U)$.

$(7) \Rightarrow(3)$ In view of the hypothesis, the restriction of $\xi_{U}$ to $P(\mathfrak{G}(U))$ is the $\operatorname{map} \xi_{U}^{1}$ : $U_{(1)} / U_{(0)} \rightarrow U_{1} / U_{0}$. The kernel of this map is $\left[U_{(1)} \cap U_{0}\right] / U_{(0)}$. Since $U$ is connected, then $U_{0}=K=U_{(0)}$ so that $U_{(1)} \cap U_{0}=U_{(1)} \cap U_{(0)}=U_{(0)}$. Hence the restriction of $\xi_{U}$ to $P(\mathfrak{G}(U))$ is injective. By Lemma $2.13, \xi_{U}$ is injective too. 
(4) $\Leftrightarrow$ (8) It is enough to prove that $U_{(n)} \cap U_{n-1}=U_{(n-1)}+\pi_{U}(V)^{n} \cap U_{n-1}$. Let $z \in U_{(n)} \cap U_{n-1}$. Then $z \in U_{(n)}=U_{(n-1)}+\pi_{U}(V)^{n}$. Hence there are $x \in U_{(n-1)}$ and $y \in$ $\pi_{U}(V)^{n}$ such that $z=x+y$. Then $y=z-x \in U_{(n)} \cap U_{n-1}+U_{(n-1)} \subseteq U_{n-1}$. We have so proved that $z=x+y \in U_{(n-1)}+\pi_{U}(V)^{n} \cap U_{n-1}$ so that $U_{(n)} \cap U_{n-1} \subseteq U_{(n-1)}+$ $\pi_{U}(V)^{n} \cap U_{n-1}$. The other inclusion is trivial.

Proposition 3.7. Let $(V, c, b)$ be a braided bialgebra and set $U:=U(V, c, b)$. Let $\left(U_{(n)}\right)_{n \in \mathbb{N}}$ be the standard filtration on $U$. Then there exists a graded braided bialgebra homomorphism $\vartheta_{U}: T(V, c) \rightarrow \mathfrak{G}(U)$ which is surjective and lifts the map $\vartheta_{U}^{1}: V \rightarrow$ $U_{(1)} / U_{(0)}=\mathfrak{G}^{1}(U): v \mapsto \pi_{U}(v)+U_{(0)}$.

Proof. Set $T:=T(V, c)$ and let $q_{n}: U_{(n)} \rightarrow U_{(n)} / U_{(n-1)}=\mathfrak{G}^{n}(U)$ denote the canonical projection. Since $\mathfrak{G}(U)$ is a connected graded coalgebra, it is clear that $\operatorname{Im}\left(\vartheta_{U}^{1}\right)=\mathfrak{G}^{1}(U) \subseteq P(\mathfrak{G}(U))$. Moreover, $\vartheta_{U}^{1}: V \rightarrow P(\mathfrak{G}(U))$ is a morphism of braided vector spaces as, for every $u, v \in V$ we have

$$
\begin{aligned}
c_{\mathfrak{G}(U)}\left(\vartheta_{U}^{1} \otimes \vartheta_{U}^{1}\right)(u \otimes v) & =c_{\mathfrak{G}(U)}\left(\left(\pi_{U}(u)+U_{(0)}\right) \otimes\left(\pi_{U}(v)+U_{(0)}\right)\right) \\
& =\left(q_{1} \otimes q_{1}\right) c_{U}\left(\pi_{U}(u) \otimes \pi_{U}(v)\right) \\
& =\left(q_{1} \otimes q_{1}\right)\left(\pi_{U} \otimes \pi_{U}\right) c_{U}(u \otimes v)=\left(\vartheta_{U}^{1} \otimes \vartheta_{U}^{1}\right) c(u \otimes v) .
\end{aligned}
$$

By the universal property of the braided tensor algebra there exists a graded braided bialgebra homomorphism $\vartheta_{U}: T(V, c) \rightarrow \mathfrak{G}(U)$ that restricted to $V$ yields $\vartheta_{U}^{1}$. Since $\mathfrak{G}(U)$ is generated as a $K$-algebra by $\mathfrak{G}^{1}(U)=\operatorname{Im}\left(\vartheta_{U}^{1}\right)$, we infer that $\vartheta_{U}$ is surjective.

Proposition 3.8. Let $(V, c, b)$ be a braided Lie algebra and set $U:=U(V, c, b)$. Let $\vartheta_{U}: T(V, c) \rightarrow \mathfrak{G}(U)$ be the map of Proposition 3.7. Then $\xi_{U} \vartheta_{U}: T(V, c) \rightarrow$ $\operatorname{gr} U$ is the unique graded braided bialgebra homomorphism lifting the map $\chi_{U}^{1}: V \rightarrow$ $U_{1} / U_{0}=: v \mapsto \pi_{U}(v)+U_{0}$. Moreover, $\xi_{U} \vartheta_{U}$ quotients to an injective braided bialgebra homomorphism $\chi_{U}: \mathcal{B}(V, c) \rightarrow \operatorname{gr} U$, i.e. (1.1) commutes.

Proof. Let $\left(U_{(n)}\right)_{n \in \mathbb{N}}$ be the standard filtration on $U$. By Proposition 3.7, $\vartheta_{U}$ : $T(V, c) \rightarrow \mathfrak{G}(U)$ is surjective and lifts the map $\vartheta_{U}^{1}$. Then $\xi_{U} \vartheta_{U}: T(V, c) \rightarrow \operatorname{gr} U$ is the unique graded braided bialgebra homomorphism from $T(V, c)$ to $\operatorname{gr} U$ which lifts the map $\chi_{U}^{1}$. Let $S^{[n]}$ be the symmetric algebra of rank $n$ of $(V, c)$ as in Definition 2.11. Now, any homogeneous primitive element of degree greater than one in $T(V, c)$ goes via $\xi_{U} \vartheta_{U}$ in a primitive element of the same degree in $\operatorname{gr} U$. Such an element is zero as non-zero primitive elements in $\operatorname{gr} U$ are concentrated in degree one (see Remark 3.5). Thus $\chi_{U}^{[0]}=\xi_{U} \pi: S^{[0]} \rightarrow$ gr $U$ quotients to a graded braided bialgebra homomorphism $\chi_{U}^{[1]}: S^{[1]} \rightarrow \operatorname{gr} U$. By the same argument, $\chi_{U}^{[1]}$ sends to zero all primitive elements of degree grater then one in $S^{[1]}$ so that $\chi_{U}^{[1]}$ quotients to $\chi_{U}^{[2]}: S^{[2]} \rightarrow \operatorname{gr} U$ and so on. Finally, one gets a graded braided bialgebra homomorphism $\chi_{U}=\chi_{U}^{[\infty]}: S^{[\infty]}=$ $\mathcal{B}(V, c) \rightarrow \operatorname{gr} U$. Note that $P(\mathcal{B}(V, c))$ identifies with $(V, c)$ via the canonical injection so that $\chi_{B}$ restricted to $P(\mathcal{B}(V, c))$ is the map $\chi_{U}^{1}$. Since $\pi_{U}(v) \in P(U)$, for all $v \in V$, we have $\operatorname{Ker}\left(\chi_{U}^{1}\right)=\operatorname{Ker}\left(\left(\pi_{U}\right)_{\mid V}\right)=0$. Thus, by Lemma 2.13, $\chi_{U}$ is injective.

Definition 3.9. Let $(V, c, b)$ be a braided bialgebra and set $U:=U(V, c, b)$. Inspired by [10, Definition, p. 316], we will say that $U$ is of PBW type whenever the canonical projection $\vartheta_{U}: T(V, c) \rightarrow \mathfrak{G}(U)$ of Proposition 3.7 quotients to a 
braided bialgebra isomorphism $\omega_{U}: \mathcal{B}(V, c) \rightarrow \mathfrak{G}(U)$ (cf. [15, p. 92] for motivating this terminology), i.e. (1.2) commutes.

Next aim is to provide a large class of braided vector spaces which give rise to universal enveloping algebras of PBW type.

Remark 3.10. Let $(V, c, b)$ be a braided Lie algebra. Assume that $(V, c)$ has combinatorial rank at most one, see Definition 2.11. Let $\beta: E(V, c) \rightarrow V$ be the restriction of $b^{[0]}$ to the space $E(V, c)$ spanned by primitive elements of $T(V, c)$ of homogeneous degree at least two. By [2, Theorem 5.10], $(V, c, \beta)$ is a braided Lie algebra in the sense of [3, Definition 4.1] and the corresponding universal enveloping algebra

$$
\mathbb{U}(V, c, \beta):=\frac{T(V, c)}{((\operatorname{Id}-\beta)[E(V, c)])}
$$

coincides with $U:=U(V, c, b)$ (the class $\mathcal{S}$, appearing in [2, Theorem 5.10], is exactly the class of braided vector spaces of combinatorial rank at most one).

THEOREM 3.11. Let $(V, c, b)$ be a braided Lie algebra. If $(V, c)$ has combinatorial rank at most one, then $U(V, c, b)$ is of $P B W$ type.

Proof. By [3, Theorem 5.4], $\mathbb{U}(V, c, \beta)$ is of PBW type in the sense of [3, Definition 4.14], i.e. the projection $\theta: S(V, c) \rightarrow \mathfrak{G}(U)$, that makes the diagram

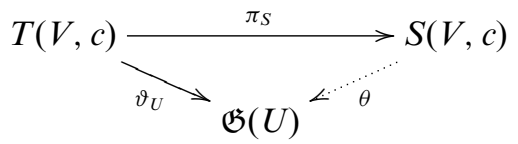

commutative, is indeed an isomorphism (here $S(V, c)=S^{[1]}$ as in Definition 2.11 and $\pi_{S}$ denotes the canonical projection). Now, since $(V, c)$ has combinatorial rank at most one, we have that $S(V, c)=\mathcal{B}(V, c)$ and $\pi_{S}=\Omega$. Thus $U$ is PBW type in the sense of Definition 3.9.

EXAMPLE 3.12. Assume char $K=0$. Let $L$ be an ordinary Lie algebra. Consider $L$ as a braided vector space through the canonical flip map $c: L \otimes L \rightarrow L \otimes L, c(x \otimes y)=$ $y \otimes x$.

By [2, Example 6.10], there exists a bracket $b$ on $(L, c)$ such that $(L, c, b)$ is a braided Lie algebra and $U(L, c, b)$ coincide with the ordinary universal enveloping algebra $U:=U(L)$. Now, since $\operatorname{char} K=0$, we have that $(V, c)$ has combinatorial rank at most one (cf. [4, Theorem 6.13]). By Theorem 3.11, $U(L, c, b)$ is of PBW type. Hence there is braided bialgebra isomorphism $\omega_{U}: \mathcal{B}(L, c) \rightarrow \mathfrak{G}(U)$ such that (1.2) commutes. Now, since $(L, c)$ has combinatorial rank at most one, we have that $\mathcal{B}(L, c)=S(L)$ (cf. [4, Remark 6.14]). Hence, the fact that $U(L, c, b)$ is of PBW type just means that the classical PBW theorem holds, see [15, p. 92].

EXAmPle 3.13. Assume $\operatorname{char} K$ is a prime number $p$. Let $V$ be a vector space regarded as a braided vector space through the canonical flip map $c$. Consider the restricted symmetric algebra of $V$ :

$$
\mathfrak{s}(V):=\frac{T(V)}{\left(x y-y x, x^{p} \mid x, y \in V\right)} .
$$


Now $\mathfrak{s}(V)$ is nothing but the restricted enveloping algebra of the trivial restricted Lie algebra $V$ so that, by [23, Theorem 6.11], we have that $P(\mathfrak{s}(V)) \cong V$. Since $\mathfrak{s}(V)$ is obtained dividing out $T(V, c)$ by elements in $E(V, c)$, by [4, Theorem 6.1 and Remark 4.3], $(V, c)$ has combinatorial rank at most one and $\mathfrak{s}(V)=\mathcal{B}(V, c)$.

Let $b$ be such that $(V, c, b)$ is a braided Lie algebra. By Remark 3.10,

$$
U(V, c, b)=\mathbb{U}(V, c, \beta)=\frac{T(V, c)}{((\operatorname{Id}-\beta)[E(V, c)])}
$$

Since $x y-y x, x^{p} \in E(V, c)$, for all $x, y \in V$, and the domain of $\beta$ is $E(V, c)$, it makes sense to set

$$
[x, y]:=\beta(x y-y x), \quad x^{[p]}:=\beta\left(x^{p}\right) .
$$

This defines two maps $[-,-]: V \otimes V \rightarrow V$ and $-^{[p]}: V \rightarrow V$. It is straightforward to check that $\left(V,[-,-],-^{[p]}\right)$ is a restricted Lie algebra, see [16, Definition 4, p. 187]. Consider the restricted enveloping algebra of $V$ :

$$
\mathfrak{u}(V):=\frac{T(V)}{\left(x y-y x-[x, y], x^{p}-x^{[p]} \mid x, y \in V\right)}
$$

Clearly there exists a projection $\lambda: \mathfrak{u}(V) \rightarrow U(V, c, b)$. Since, by [23, Theorem 6.11], we have $P(\mathfrak{u}(V)) \cong V$, we can apply Lemma 2.13 to conclude that $\lambda$ is bijective whence $\mathfrak{u}(V)=U(V, c, b)$.

Conversely, let $[-,-]: V \otimes V \rightarrow V$ and $-^{[p]}: V \rightarrow V$ be such that $\left(V,[-,-],-^{[p]}\right)$ is a restricted Lie algebra and set $A:=\mathfrak{u}(V)$. This is an ordinary Hopf algebra, see [24, p. 23]. In particular, it is a braided bialgebra with braiding the canonical flip map on $A$. It is primitively generated as, by construction, it is generated by the image of $V$ in $A$. By Remark 2.9, $A \cong U\left(P, c_{P}, b_{P}\right)$ where $\left(P, c_{P}, b_{P}\right)$ is the infinitesimal braided Lie algebra of $A$.

Now the canonical map $\sigma: V \rightarrow P=P(A)$ is bijective (cf. [23, Theorem 6.11]). Since $c_{P}$ is the restriction of the braiding of $A$, then $c_{P}$ is the canonical flip map on $P$. Thus $\sigma:(V, c) \rightarrow\left(P, c_{P}\right)$ is an isomorphism of braided vector spaces, where $c$ is the canonical flip map on $V$. Hence we can endow $(V, c)$ with a bracket $b$ such that $(V, c, b)$ is a braided Lie algebra and $\sigma:(V, c, b) \rightarrow\left(P, c_{P}, b_{P}\right)$ is an isomorphism of braided Lie algebras. Hence $A \cong U:=U(V, c, b)$. By the foregoing, $(V, c)$ has combinatorial rank at most one so that, by Theorem 3.11, $U$ is of PBW type. Hence there is a braided bialgebra isomorphism $\omega_{U}: \mathcal{B}(V, c) \rightarrow \mathfrak{G}(U)$ such that (1.2) commutes. Since, by the initial part, one has that $\mathcal{B}(V, c)=\mathfrak{s}(V)$, then we get an isomorphism $\omega_{U}$ : $\mathfrak{s}(V) \rightarrow \mathfrak{G}(\mathfrak{u}(V))$ which is a PBW theorem for restricted enveloping algebras, see [23, Proposition 6.12].

4. Basis for the universal enveloping algebra. In this section, we will discuss the problem of determining a basis for a universal enveloping algebra of PBW type. 
Lemma 4.1. Let $A, B, C$ be vector spaces, and let $W \leq A$ and $B^{\prime} \leq B$ be two vector subspaces. Let $f, g, p$ and $q$ be K-linear maps as in the following commutative diagram:

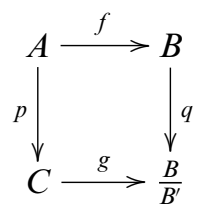

where $q$ is the canonical projection. If $g$ and $\left.p\right|_{W}: W \rightarrow$ C are isomorphisms then $f(W) \oplus$ $B^{\prime}=B$. Moreover, $f_{\mid W}$ is injective.

Proof. The first part of the statement is exactly [6, Lemma 4.21]. Let $w \in W \cap \operatorname{Ker} f$. Then $g p(w)=q f(w)=0$. Since $g$ is bijective, we get $p(w)=0$. Since $\left.p\right|_{W}: W \rightarrow C$ is an isomorphism, we conclude that $w=0$.

Proposition 4.2. Let $(V, c, b)$ be a braided Lie algebra and set $U:=U(V, c, b)$. Let $\Omega: T(V, c) \rightarrow \mathcal{B}(V, c)$ be the canonical projection and denote by $\Omega^{n}: V^{\otimes n} \rightarrow \mathcal{B}(V, c)^{n}$ the nth graded component of $\Omega$. Assume that $U$ is of $P B W$ type and let $W_{n}$ be a vector subspace of $V^{\otimes n}$ such that $\Omega_{\mid W_{n}}^{n}$ is an isomorphism. Then $U_{(n)}=U_{(n-1)} \oplus \pi_{U}\left(W_{n}\right)$, where $\left(U_{(n)}\right)_{n \in \mathbb{N}}$ is the standard filtration on $U$. Moreover, $\pi_{U \mid W_{n}}$ is injective.

Proof. Clearly, $\pi_{U}\left(V^{\otimes n}\right) \subseteq U_{(n)}$ so $\pi_{U}$ induces a map $\tilde{\pi}^{n}: V^{\otimes n} \rightarrow U_{(n)}$. Apply Lemma 4.1 to the following diagram

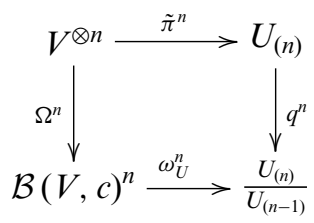

where $q^{n}: U_{(n)} \rightarrow U_{(n)} / U_{(n-1)}$ is the canonical projection and $\omega_{U}^{n}$ is the $n$th graded component of the isomorphism $\omega_{U}: \mathcal{B}(V, c) \rightarrow \mathfrak{G}(U)$ of Definition 3.9.

REMARK 4.3. Let $(V, c, b)$ be a braided Lie algebra and set $U:=U(V, c, b)$. Assume that $U$ is of PBW type. Set $\Theta:=\left\{n \in \mathbb{N} \mid \mathcal{B}(V, c)^{n} \neq\{0\}\right\}$ and suppose that, for each $n \in \Theta$, we can find a linearly independent set $Z_{n}:=\left\{v_{n, i} \mid i \in I_{n}\right\}$ consisting of elements of $V^{\otimes n}$ with the property that $W_{n}:=\operatorname{Span}_{K} Z_{n}$ is such that $\Omega_{\mid W_{n}}^{n}$ is an isomorphism. By Proposition 4.2, $U_{(n)}=U_{(n-1)} \oplus \pi_{U}\left(W_{n}\right)$. Moreover, $\pi_{U \mid W_{n}}$ is injective. Therefore, $\left\{\pi_{U}\left(v_{n, i}\right) \mid n \in \Theta, i \in I_{n}\right\}$ is a basis for $U$. Hence, as in the classical case, finding a basis for $U$ reduces to determine a suitable basis $\left\{v_{n, i} \mid n \in \Theta, i \in I_{n}\right\}$ for $\mathcal{B}(V, c)$ as above. Results in this direction are obtained by Kharchenko (cf. [18, Theorem 2]) and, more generally, by Ufer (cf. [30, Theorem 34]) when $(V, c)$ is a braided vector space of diagonal type or left triangular respectively.

Next aim is show how the computation of a PBW basis for the classical universal enveloping algebra and for the restricted enveloping algebra fits into the theory above.

EXAmple 4.4. Assume $\operatorname{char} K=0$. Let $L$ be an ordinary Lie algebra which is assumed to have a totally ordered basis $(X, \leq)$. By Example 3.12 , the universal enveloping algebra $U:=U(L)$ can be identified with the universal enveloping algebra 
$U(L, c, b)$ where $c$ is the canonical flip map on $L$ and $b$ a suitable bracket for $(L, c)$. Moreover, $(L, c)$ has combinatorial rank at most one and $\mathcal{B}(L, c)$ is the ordinary symmetric algebra $S(L)$. Consider the canonical projection $\Omega: T(L) \rightarrow S(L)$. For $n=0$, set $Z_{0}:=\left\{1_{K}\right\}$ and, for $n \geq 1$, let $Z_{n} \subseteq L^{\otimes n}$ be the set

$$
\left\{x_{1} \cdot{ }^{\prime} \cdots T_{T} x_{n} \mid x_{i} \in X, \forall 1 \leq i \leq n \text { and } x_{1} \leq x_{2} \leq \cdots \leq x_{n}\right\} .
$$

If we set $W_{n}:=\operatorname{Span}_{K} Z_{n}$, it is clear that $\Omega_{\mid W_{n}}^{n}$ is an isomorphism. Hence, by Remark 4.3, The elements $x_{1} \cdot{ }_{U} \cdots U_{U} x_{n}$, where $n \geq 1, x_{i} \in X$, for all $1 \leq i \leq n$, and $x_{1} \leq x_{2} \leq$ $\cdots \leq x_{n}$, along with $1_{K}$, form a basis of the universal enveloping algebra $U(L)$ of $L$. This theorem is due to Poincarî, Birkhoff and Witt and the basis is called the PBW basis of the universal enveloping algebra (see e.g. [15, Corollary C, p. 92]).

REMARK 4.5. Keep the assumptions and notations of Example 4.4. Observe that proving the isomorphism $U(L) \cong U(L, c, b)$ requires the condition $P U(L) \cong L$ (cf. [2, Example 6.10]). One could object that, in order to check this isomorphism, a basis of $U(L)$ is needed. We can clear the hurdle as follows. By [15, p. 92], $U(L)$ fulfills the PBW theorem. Now, mimicking the proof of [3, Corollary 5.5], we arrive at $P U(L) \cong L$ without using a basis of $U(L)$.

EXAMPLE 4.6. Assume $\operatorname{char} K$ is a prime number $p$. Let $\left(L,[-,-],-^{[p]}\right)$ be a restricted Lie algebra which is assumed to have a totally ordered basis $(X, \leq)$. By Example 3.13, the restricted enveloping algebra $\mathfrak{u}:=\mathfrak{u}(L)$ can be identified with the universal enveloping algebra $U(L, c, b)$ where $c$ is the canonical flip map on $L$ and $b$ a suitable bracket for $(L, c)$. Moreover, $(L, c)$ has combinatorial rank at most one. Hence by Theorem 3.11, $U(L, c, b)$ is of PBW type. Furthermore, by Example 3.13, $\mathcal{B}(L, c)$ is the restricted symmetric algebra $\mathfrak{s}(L)$. Consider the canonical projection $\Omega: T(L) \rightarrow \mathfrak{s}(L)$. For $n=0$, set $Z_{0}:=\left\{1_{K}\right\}$ and, for $n \geq 1$, let $Z_{n} \subseteq L^{\otimes n}$ be the set

$$
\begin{aligned}
& \left\{x_{1}^{t_{1}} \cdot{ }_{T} \cdots x_{T} x_{n}^{t_{n}} \mid x_{i} \in X, 0 \leq t_{i} \leq p-1, \forall 1 \leq i \leq n, t_{1}+\cdots+t_{n}\right. \\
& \left.\quad=n \text { and } x_{1}<x_{2}<\cdots<x_{n}\right\} .
\end{aligned}
$$

Then $Z_{n}=\emptyset$ whenever $\mathcal{B}(L, c)^{n}=\{0\}$ and $Z_{n}$ is linearly independent otherwise. If we set $W_{n}:=\operatorname{Span}_{K} Z_{n}$, it is clear that $\Omega_{\mid W_{n}}^{n}$ is an isomorphism for all $n \in \mathbb{N}$. Hence, by Remark 4.3, The elements $x_{1}^{t_{1}} \cdot \mathfrak{u} \cdots \mathfrak{u}_{\mathfrak{u}} x_{n}^{t_{n}}$, where $n \geq 1, x_{i} \in X, 0 \leq t_{i} \leq p-1$, for all $1 \leq i \leq n$, and $x_{1}<x_{2}<\cdots<x_{n}$, along with $1_{K}$, form a basis of $\mathfrak{u}(L)$. This basis is called the PBW basis of the restricted enveloping algebra (see e.g. [24, p. 23]).

We now give an example of a PBW basis for the universal enveloping algebra of a braided Lie algebra whose bracket $c$ is not a symmetry, i.e. $c^{2} \neq \mathrm{Id}$.

ExAmple 4.7. Assume char $K=0$. Let $V:=K x_{1} \oplus K x_{2}$ and define a diagonal braiding $c$ on $V$ by setting $c\left(x_{i} \otimes x_{j}\right)=q_{i j} x_{j} \otimes x_{i}$, where $q_{11}=\gamma \in K$ and $q_{i j}=1$ for all $(i, j) \neq(1,1)$. Assume $\gamma$ is not a root of unity. In view of [3, Example 9.8], the endomorphism $c$ has minimal polynomial $(X-\gamma)\left(X^{2}-1\right)$ (whence it is not a symmetry), the braided vector space $(V, c)$ has combinatorial rank at most one and Nichols algebra $\mathcal{B}(V, c)=T(V, c) /\left(x_{2} x_{1}-x_{1} x_{2}\right)$. Consider the braided bialgebra $A:=T(V, c) /\left(x_{2} x_{1}-x_{1} x_{2}-x_{1}\right)$. Still by [3, Example 9.8], we know that the infinitesimal part of $A$ identifies with $(V, c)$. Thus, by Remark 2.9, there is a braided bracket $b$ on $(V, c)$ such that $(V, c, b)$ is a braided Lie algebra and $A \cong U(V, c, b)$. By Theorem 3.11, $U(V, c, b)$ is of PBW type. 
For all $n \in \mathbb{N}$, set $Z_{n}:=\left\{x_{1}^{t_{1}} \cdot T x_{2}^{t_{2}} \mid t_{1}, t_{2} \in \mathbb{N}, t_{1}+t_{2}=n\right\} \subseteq V^{\otimes n}$. If we set $W_{n}:=$ $\operatorname{Span}_{K} Z_{n}$, it is clear that $\Omega_{\mid W_{n}}^{n}$ is an isomorphism for all $n \in \mathbb{N}$. Hence, by Remark 4.3, The elements $x_{1}^{t_{1}} \cdot A x_{2}^{t_{2}}$, where $t_{1}, t_{2} \in \mathbb{N}$, form a basis of $A$. It is remarkable that $A$ is not a classical universal enveloping algebra as its infinitesimal braiding is not a symmetry. Note also that, as we will see in Theorem 5.2, since $U(V, c, b)$ is of PBW type then $U$ is strictly generated by $V$. Hence the $n$th term of coradical filtration of $A$ is $\sum_{i=0}^{n} \pi_{A}(V)^{i}$, where $\pi_{A}: T(V, c) \rightarrow A$ denotes the canonical projection. Moreover, by Theorem 6.2, $A$ is what we will call a braided bialgebra lifting of $\mathcal{B}(V, c)$.

5. Cosymmetric. We will investigate universal enveloping algebras which are cosymmetric in the sense of [20, Definition 3.1].

Definition 5.1. Let $\left(C, \Delta_{C}, \varepsilon_{C}, c\right)$ be a connected braided coalgebra. In view of [5, Remark 1.12], the braiding of $C$ induces a braiding $c_{P}$ on the space $P:=P(C)$ of primitive elements in $C$. Moreover, $\left(P, c_{P}\right)$ is a braided vector space. Let $1_{C}$ be the unique group-like element of $C$. Let $\phi: C \rightarrow C$ be defined by $\phi(c):=c-\varepsilon_{C}(c) 1_{C}$ and let $\Delta_{C}^{n}: C \rightarrow C^{\otimes(n+1)}$ be the $n$th iterated comultiplication of $C$. For $n \in \mathbb{N}$, let $C_{n}$ be the $n$th term of the coradical filtration of $C$. By [20, Lemma 2.2], which is deduced from [28, Proposition 11.0.5], we have $\operatorname{Ker}\left(\phi^{\otimes(n+1)} \Delta_{C}^{n}\right)=C_{n}$, for all $n \in \mathbb{N}$. Moreover, $\phi^{\otimes(n+1)} \Delta_{C}^{n}\left(C_{n+1}\right) \subseteq P^{\otimes(n+1)}$ so that, for all $n>0$, the restriction of $\phi^{\otimes n} \Delta_{C}^{n-1}$ quotients to a map $\mu_{n}: C_{n} / C_{n-1} \rightarrow P^{\otimes n}$. Set $\mu_{0}:=\operatorname{Id}_{K}$. Then $\mu_{C}:=\bigoplus_{n \in \mathbb{N}} \mu_{n}: \operatorname{gr} C \rightarrow T^{c}\left(P, c_{P}\right)$ is called the linearisation map and it is an injective coalgebra map. Here $T^{c}\left(P, c_{P}\right)$ denotes the braided cotensor coalgebra of $\left(P, c_{P}\right)$ also known as quantum shuffle algebra and denoted by $\operatorname{Sh}_{c P}(P)$. The linearisation map can equivalently be constructed by means of the universal property of the cotensor coalgebra $T^{c}(P)$ [28, Proposition 12.1.1]. Following [20, Definition 3.1], we will say that a connected braided bialgebra $C$ is cosymmetric if $\operatorname{Im}\left(\mu_{C}\right) \subseteq \mathcal{B}(V, c)$ (note that in [20], the Nichols algebra $\mathcal{B}(V, c)$ is denoted by $\left.S_{c}(V)\right)$.

For a connected braided bialgebra $B$, one has that the linearisation map $\mu_{B}$ : $\operatorname{gr} B \rightarrow T^{c}\left(P, c_{P}\right)$ is indeed a braided bialgebra map (cf. [20, Proposition 3.3]).

THEOREM 5.2. Let $(V, c, b)$ be a braided Lie algebra and set $U:=U(V, c, b)$. The following assertions are equivalent:

(1) $U$ is of PBW type.

(2) $U$ is cosymmetric.

(3) $U$ is strictly generated by $V$.

(4) $\operatorname{gr} U$ is primitively generated.

(5) The map $\chi_{U}: \mathcal{B}(V, c) \rightarrow \operatorname{gr} U$ of Proposition 3.8 is bijective.

Proof. By Remark 2.7, the primitively generated braided bialgebra $U$ is indeed a braided Hopf algebra. Hence, the equivalence between (2), (3), (4) and (5) follows by [20, Theorem 3.5].

Let $\left(U_{(n)}\right)_{n \in \mathbb{N}}$ be the standard filtration on $U$. Let $\vartheta_{U}: T(V, c) \rightarrow \mathfrak{G}(U)$ be the graded braided bialgebra homomorphism of Proposition 3.7.

$(1) \Rightarrow(3)$ By hypothesis, $\vartheta_{U}$ quotients to a braided bialgebra isomorphism $\omega_{U}: \mathcal{B}(V, c) \rightarrow \mathfrak{G}(U)$. We have $P(\mathfrak{G}(U))=\omega_{U}(P(\mathcal{B}(V, c)))=\omega_{U}(V)=U_{(1)} / U_{(0)}$. By Proposition 3.6, we get that $U$ is strictly generated by $V$.

(5) $\Rightarrow$ (1) By Proposition 3.8, we have $\chi_{U} \Omega=\xi_{U} \vartheta_{U}$. Since both $\chi_{U}$ and $\Omega$ are surjective, so is $\xi_{U}$. By Proposition 3.6, $\xi_{U}$ is bijective. Set $\omega_{U}:=\left(\xi_{U}\right)^{-1} \chi_{U}$. 
REMARK 5.3. In Theorem 3.11, we gave a class of braided vector spaces such that $U(V, c, b)$ is of PBW type whatever is the bracket. It is still an open question whether the property of $U(V, c, b)$ to be of PBW type depends just on the braided vector space $(V, c)$ in general. In Example 5.6, we will exhibit a braided Lie algebra $(V, c, b)$ such that $U(V, c, b)$ is not of PBW type.

THEOREM 5.4. Let $(V, c, b)$ be a braided Lie algebra and set $U:=U(V, c, b)$. Assume $U^{[n]}$ is cosymmetric for some $n \in \mathbb{N}$. Then $U^{[n+1]}$ is cosymmetric and $U(V, c, b)=U^{[n+1]}$.

Proof. By construction (cf. the proof of [2, Proposition 4.5]), we have that, for all $n \in \mathbb{N}, K^{[n]}:=\operatorname{Im}\left(\operatorname{Id}_{P^{[n]}}-i^{[n]} b^{[n]}\right)$ is a categorical subspace of $P^{[n]}$. For all $n \in \mathbb{N}$, set $W^{[n]}:=\operatorname{Ker}\left(\pi_{n}^{n+1}\right) \cap P^{[n]}$

By Lemma 2.12, $W^{[n]}=K^{[n]}$ and $P^{[n]}=W^{[n]} \oplus V^{[n]}$, for all $n \in \mathbb{N}$. Assume now that $U^{[n]}$ is cosymmetric for a fixed $n \in \mathbb{N}$. By [20, Lemmata 4.2 and 4.4], taking $W^{[n]}$ as $W$ and $V^{[n]}$ as $W^{\prime}$, we get that $U^{[n+1]}$ is cosymmetric and $P^{[n+1]}=\pi_{n}^{n+1}\left(P^{[n]}\right)$. Now

$$
P^{[n+1]}=\pi_{n}^{n+1}\left(P^{[n]}\right)=\pi_{n}^{n+1} i^{[n]} b^{[n]}\left(P^{[n]}\right)=i^{[n+1]} b^{[n]}\left(P^{[n]}\right)=\operatorname{Im}\left(i^{[n+1]}\right)=V^{[n+1]} .
$$

Hence $W^{[n+1]}=0$. Now, by definition of $\pi_{n+1}^{n+2}$, we have $\operatorname{Ker}\left(\pi_{n+1}^{n+2}\right)=\left(K^{[n+1]}\right)$ so that, by the foregoing, we get $\operatorname{Ker}\left(\pi_{n+1}^{n+2}\right)=\left(W^{[n+1]}\right)=0$. Therefore, $\pi_{n+1}^{n+2}$ is bijective and $U^{[n+1]}=U^{[n+2]}$. Thus $U(V, c, b)=\underline{\lim } U^{[i]}=U^{[n+1]}$.

COROLlaRY 5.5. Let $(V, c)$ be a braided vector space. If there is $n \in \mathbb{N}$ such that the symmetric algebra $S^{[n]}$ of rank $n$ is cosymmetric, then $(V, c)$ has combinatorial rank at most $n+1$ in the sense of Definition 2.11.

Proof. Set $U:=U\left(V, c, b_{t r}\right)$ where $b_{t r}$ is the trivial bracket on $(V, c)$. Note that $U^{[t]}=S^{[t]}$ is the symmetric algebra of rank $t$ for all $t \in \mathbb{N}$. Hence, by Theorem 5.4, we have that $U^{[n+1]}$ is cosymmetric and $\mathcal{B}(V, c)=U\left(V, c, b_{t r}\right)=U^{[n+1]}$. This means $(V, c)$ has combinatorial at most $n+1$.

EXAMPLE 5.6. At the end of [19], an example of a two-dimensional braided vector space $(V, c)$ of combinatorial rank 2 is given. The braiding $c$ is of diagonal type of the form $c\left(x_{i} \otimes x_{j}\right)=q_{i, j} x_{j} \otimes x_{i}, 1 \leq i, j \leq 2$, where $x_{1}, x_{2}$ forms a basis of $V$ over $K$, $q_{1,2}=1 \neq-1$ and $q_{i, j}=-1$ for all $(i, j) \neq(1,2)$. By Corollary 5.5, applied to the case $n=0, T:=T(V, c)$ is not cosymmetric.

Since $T$ is a primitively generated braided bialgebra, by Remark 2.9, we know that $T$ coincides with the universal enveloping algebra of its infinitesimal braided Lie algebra $\left(P, c_{P}, b_{P}\right)$. We have so exhibited a braided Lie algebra $\left(P, c_{P}, b_{P}\right)$ such that $U\left(P, c_{P}, b_{P}\right)$ is not cosymmetric. By Theorem 5.2, $U\left(P, c_{P}, b_{P}\right)$ is not of PBW type.

6. Lifting of Nichols algebras. In this section, we investigate braided bialgebra liftings of Nichols algebras. We will characterise them in terms of universal enveloping algebras of PBW type.

Definition 6.1. Let $(V, c)$ be a braided vector space. We will say that a braided bialgebra $B$ is a lifting of $\mathcal{B}(V, c)$ if there is a graded braided bialgebra isomorphism $\chi_{B}: \mathcal{B}(V, c) \rightarrow \operatorname{gr} B$. 
THEOREM 6.2. Let $(V, c)$ be a braided vector space and let $B$ be a braided bialgebra. The following assertions are equivalent:

(1) $B$ is a lifting of $\mathcal{B}(V, c)$.

(2) There is a bracket $b$ on $(V, c)$ such that $(V, c, b)$ is a braided Lie algebra, $U(V, c, b)$ is of $P B W$ type and $B \cong U(V, c, b)$ as braided bialgebras.

Proof. (1) $\Rightarrow$ (2) By hypothesis, there is a braided bialgebra isomorphism $\chi_{B}$ : $\mathcal{B}(V, c) \rightarrow \operatorname{gr} B$. Since $\mathcal{B}(V, c)$ is primitively generated, the same holds for $\operatorname{gr} B$ whence for $B$. By Remark 2.9, $B \cong U\left(P, c_{P}, b_{P}\right)$, where $\left(P, c_{P}, b_{P}\right)$ is the infinitesimal braided Lie algebra of $B$. Clearly $\chi_{B}$ preserves primitive elements so that it induces an isomorphism of braided vector spaces $\gamma:(V, c) \rightarrow\left(P, c_{P}\right)$. Since $\gamma$ is bijective, there is a bracket $b$ on $(V, c)$ such that $(V, c, b)$ is a braided Lie algebra and $U(V, c, b) \cong U\left(P, c_{P}, b_{P}\right) \cong B$. Set $U:=U(V, c, b)$ and denote by $\varphi: U \rightarrow B$ this isomorphism. Then $\varphi_{\mid V}=\gamma$. Thus one easily checks that the diagram

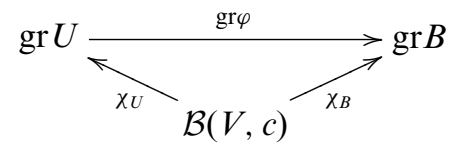

commutes. Since both $\chi_{B}$ and $\varphi$ are isomorphisms, we get that $\chi_{U}$ is an isomorphism too. By Theorem 5.2, we get that $U$ is of PBW type.

(2) $\Rightarrow$ (1) Since $U:=U(V, c, b)$ is of PBW type, by Theorem 5.2 we have that the map $\chi_{U}: \mathcal{B}(V, c) \rightarrow \operatorname{gr} U$ of Proposition 3.8 is bijective. Hence $U$ is a lifting of $\mathcal{B}(V, c)$.

In view of Theorem 6.2 , given a braided vector space $(V, c)$, studying braided bialgebra liftings of $\mathcal{B}(V, c)$ amounts to investigate braided brackets $b$ on $(V, c)$ such that $(V, c, b)$ is a braided Lie algebra and $U(V, c, b)$ is of PBW type.

COROLlary 6.3. Let $(V, c)$ be a braided vector space of combinatorial rank at most one and let $B$ be a braided bialgebra. The following assertions are equivalent.

(1) $B$ is a lifting of $\mathcal{B}(V, c)$.

(2) There is a bracket $b$ on $(V, c)$ such that $(V, c, b)$ is a braided Lie algebra and $B \cong U(V, c, b)$ as braided bialgebras.

Proof. It follows by Theorem 6.2 and Theorem 3.11.

AcKNOWLEDGMENTS. We would like to thank the referee for several useful suggestions that improved an earlier version of this paper.

\section{REFERENCES} 582.

1. A. Ardizzoni, Separable functors and formal smoothness, J. K-theory 1(3) (2008), 535-

2. A. Ardizzoni, A Milnor-Moore type theorem for primitively generated braided bialgebras, J. Algebra 327(1) (2011), 337-365.

3. A. Ardizzoni, On primitively generated braided Bialgebras, Algebr. Represent. Theory, to appear (doi:10.1007/s10468-010-9257-z).

4. A. Ardizzoni, On the combinatorial rank of a graded braided bialgebra, J. Pure Appl. Algebra 215 (2011), 2043-2054.

5. A. Ardizzoni, C. Menini and D. Ştefan, Braided bialgebras of Hecke-type, J. Algebra 321(3) (2009), 847-865. 
6. A. Ardizzoni, C. Menini and D. Ştefan, PBW deformations of braided symmetric algebras and a Milnor-Moore type theorem for braided bialgebras, preprint (arXiv:math.QA/0604181v2).

7. A. Ardizzoni and F. Stumbo, Quadratic Lie algebras, Commun. Algebra, to appear.

8. J. C. Baez, Hochschild homology in a braided tensor category, Trans. Amer. Math. Soc. 334(2) (1994), 885-906.

9. R. Berger, The quantum Poincaré-Birkhoff-Witt theorem. Commun. Math. Phys. 143(2) (1992), 215-234.

10. S. Braverman and D. Gaitsgory, Poincaré-Birkhoff-Witt theorem for quadratic algebras of Koszul type, J. Algebra 181(2) (1996), 315-328.

11. X. Gomez and S. Majid, Braided Lie algebras and bicovariant differential calculi over co-quasitriangular Hopf algebras, J. Algebra 261(2) (2003), 334-388.

12. D. I. Gurevich, Generalized translation operators on Lie groups. (Russian. English, Armenian summary) Izv. Akad. Nauk Armyan. SSR Ser. Mat. 18(4) (1983), 305-317.

13. D. Gurevich, Hecke symmetries and braided Lie algebras. in Spinors, twistors, Clifford algebras and quantum deformations (Sobótka Castle, 1992), 317-326, Fund. Theories Phys., Vol. 52 (Kluwer Academic Publishers, Dordrecht, 1993).

14. M. Helbig, A PBW basis criterion for pointed Hopf algebras, preprint (arXiv:1011.0140v1).

15. J. E. Humphreys, Introduction to Lie algebras and representation theory, in Graduate texts in mathematics, Vol. 9 (Springer-Verlag, New York-Berlin, 1972).

16. N. Jacobson, Lie algebras, in Interscience tracts in pure and applied mathematics, No. 10 (Interscience Publishers (a division of John Wiley \& Sons), New York-London, 1962).

17. V. K. Kharchenko, An algebra of skew primitive elements, Algebra Logic 37(2) (1998), $101-126$.

18. V. K. Kharchenko, A quantum analogue of the Poincaré-Birkhoff-Witt theorem. Algebra and Logic 38(4) (1999), 259-276.

19. V. K. Kharchenko, Skew primitive elements in Hopf algebras and related identities, J. Algebra 238(2) (2001), 534-559.

20. V. K. Kharchenko, Connected braided Hopf algebras, J. Algebra 307(1) (2007), 24-48.

21. G. Lusztig, Canonical bases arising from quantized enveloping algebras, J. Amer. Math. Soc. 3(2) (1990), 447-498.

22. S. Majid, Quantum and braided-Lie algebras, J. Geom. Phys. 13(4) (1994), 307-356.

23. J. W. Milnor and J. C. Moore, On the structure of Hopf algebras, Ann. Math. 81(2) (1965), 211-264.

24. S. Montgomery, Hopf Algebras and their actions on rings, CMBS Region. Conf. Ser. Math. 82 (1993).

25. B. Pareigis, On Lie algebras in the category of Yetter-Drinfeld modules, Appl. Categ. Struct. 6(2) (1998), 151-175.

26. C. M. Ringel, PBW-bases of quantum groups, J. Reine Angew. Math. 470 (1996), 51-88.

27. M. Rosso, An analogue of P.B.W. theorem and the universal $R \mathrm{R}$-matrix for $U_{h} \mathrm{sl}(N+1)$, Commun. Math. Phys. 124(2) (1989), 307-318.

28. M. Sweedler, Hopf algebras (Benjamin, New York, 1969).

29. M. Takeuchi, Survey of braided Hopf algebras, in New trends in Hopf algebra theory (La Falda, 1999), pp. 301-323; Contemp. Math., Vol. 267 (Amer. Math. Soc., Providence, RI, 2000).

30. S. Ufer, PBW bases for a class of braided Hopf algebras, J. Algebra 280(1) (2004), 84-119. 1156.

31. M. Wambst, Complexes de Koszul quantiques, Ann. Inst. Fourier 43(4) (1993), 1089

32. S. L. Woronowicz, Differential calculus on compact matrix pseudogroups (quantum groups), Commun. Math. Phys. 122(1) (1989), 125-170.

33. H. Yamane, A Poincare-Birkhoff-Witt theorem for the quantum group of type $A_{N}$. Proc. Japan Acad. Ser. A Math. Sci. 64(10) (1988), 385-386. 